\title{
Platinum Tunneling Morphology in a Zeolite Catalyst
}

M. R. Kupsta*, G. R. D. Elliott**, V. A. Munoz**, C. Fairbridge**, J.-P. Charland*** and R. J. Mikula**

*National Institute for Nanotechnology, 11421 Saskatchewan Drive Edmonton, Alberta, Canada, T6G 2M9

**CanmetENERGY, Natural Resources Canada, 1 Oil Patch Drive Devon, AB Canada, T9G 1A8

*** CanmetENERGY, Natural Resources Canada, 1 Haanal Drive, Ottawa ON K1A 1M1

The production of upgraded crude oil from oil sands requires an increase in the hydrogen to carbon ratio in the heavy hydrocarbons. This is done commercially by both removing carbon by coking and adding hydrogen by hydrocracking and hydrogenation. Coking is a chemically complex thermal process that cracks some large molecules to form free radicals and many of these polymerize to form coke. Hydrocracking occurs in the presence of catalyst to increase the $\mathrm{H} / \mathrm{C}$ ratio producing lighter hydrocarbons of higher commercial value. The efficiency of the hydroprocessing catalyst is affected by contaminants, such as nitrogen and sulphur, which remain in the organic phase poisoning the active catalyst sites.

In bitumen upgrading applications, the mesopore distribution in the catalysts is important in controlling access of large hydrocarbon molecules to active sites [1]. At the same time, nano-pore size distribution control would help to design catalysts that could allow hydrogen access to the active sites, but not the larger sulphur containing molecules. Pt-silicalites were reacted under controlled conditions in a simulated exhaust gas stream at $800^{\circ} \mathrm{C}$. The acidic species created by exhaust gas reacting at the platinum/zeolite interface allowed the platinum to burrow into the zeolite $[2,3]$. Control of the reaction gas composition and the platinum distribution can allow for control of the size and distribution of mesopore tunnels in the catalyst substrate giving rise to active hydrocracking catalysts. However, the three dimensional morphology of the tunnel organization by the platinum within the catalyst is unknown. A successful three dimensional reconstruction will allow us to describe the complex network of the tunnels, and ultimately help in modelling the catalyst performance.

We report on 3D reconstruction of platinum tunneling patterns in zeolite catalysts particles by mill and monitoring in a Hitachi NB 5000 FIB/SEM dual beam instrument (FIG. 1). This study was aimed to reconstruct the platinum tunneling patterns in the zeolite catalyst by milling, image capture, image processing and tomographic reconstruction of the milled slices. Catalyst particles were coated with tungsten in situ using the gas injection system. This conductive layer secures the particle to the aluminum stub to avoid particle movement during the milling process and minimize charging artifacts. Milling of the particle was carried out using the $\mathrm{Ga}^{+}$FIB column while imaging was done by using the Schottky SEM column. The secondary electron signal was acquired using an Everhart-Thornley detector. The mill and monitor recipe involved successively removing $5 \mathrm{~nm}$ and acquiring 400 to 600 SEM images, one after every milling step, to capture the whole catalyst particle. Each SEM image of the milled catalyst was acquired using frame integration over 128 frames. The SEM images seen in FIG. 1 were used in Fiji [4], an image processing program, to be better suited for 3D reconstruction. Image processing included cropping, feature enhancement, and localized thresholding to emphasize the features in the catalyst particle. The processed image stack 
was assembled using software from the TEMography software package [5]. Stack N Viz was used to import and align SEM images while Visualizer was used to carry out the 3D reconstruction shown in FIG. 2. The data provided from the 3D reconstruction allows for volumetric data of the tunnels in the particle, lengths and branching information. Preliminary data shows that the tunnels occupy approximately $1.5 \%$ of the particle volume.

\section{References:}

[1] Chen et al., Catalysis Today. 125 (2007) 256.

[2] Yang et al., Microporous and Mesoporous Materials. 117 (2009) 33.

[3] Kato et al, Angew. Chem. Int. Ed. 43, 1251, 2004

[4] http://pacific.mpi-cbg.de/

[5] https://www.temography.com/modules/home1/

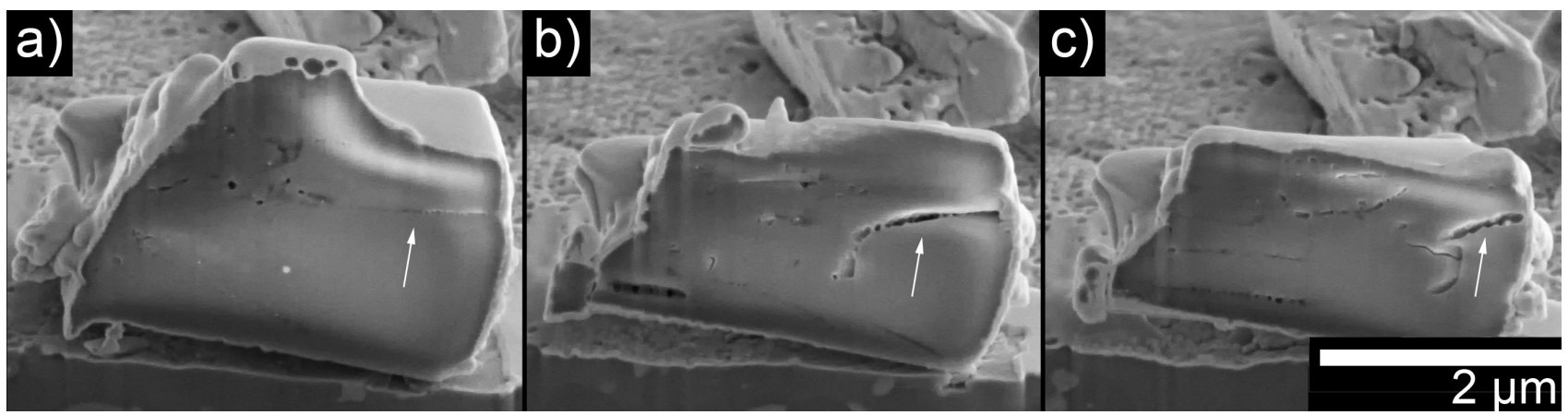

FIG. 1. SEM images of the catalyst acquired at 3 locations during the mill and monitor processes were shown: a) $0 \mathrm{~nm}$ (start), b) $25 \mathrm{~nm}$ and c) $50 \mathrm{~nm}$. Slicing through the catalyst revealed a complex network of tunnels created by the platinum burrowing.
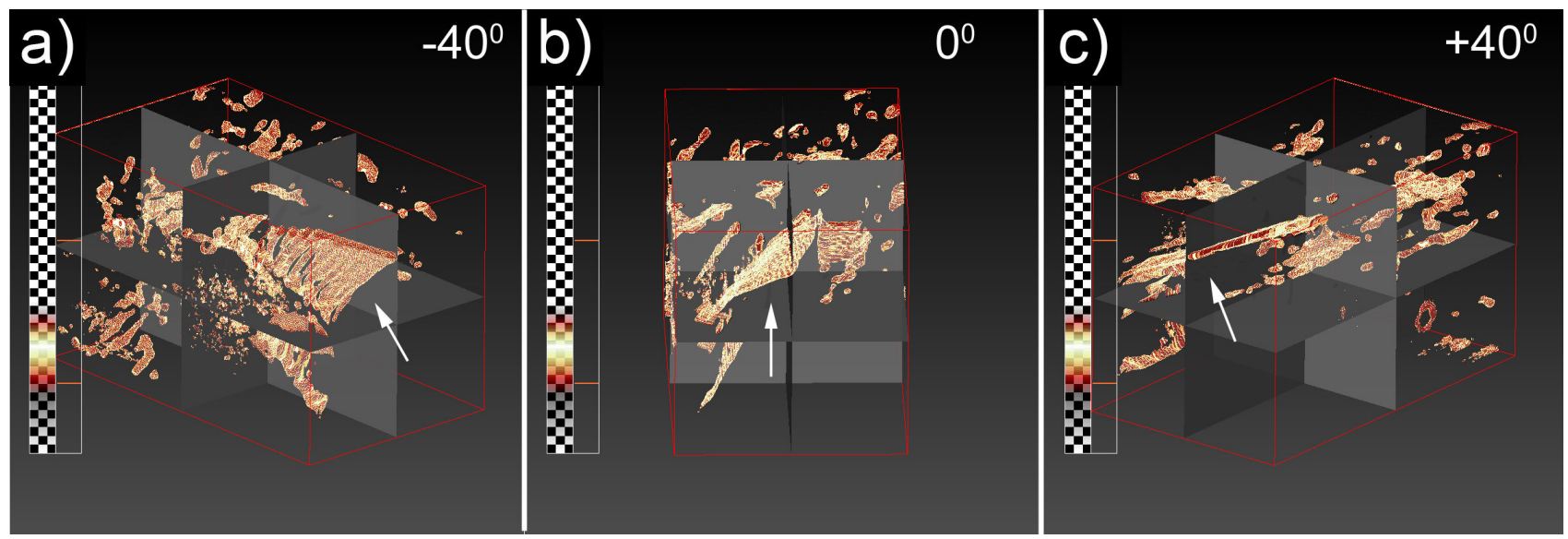

FIG. 2. A 3D reconstruction of catalyst in FIG. 1, using TEMography software, was shown at three different orientations. The tunnel depicted in Figure 1 on the right side of the particle was visible in the quadrants closest in this figure. The images were rotated to give the viewer a better spatial awareness of the reconstructed features: a) $40^{\circ}$ counter clockwise, b) $0^{\circ}$ and c) $40^{\circ}$ clockwise. 